\title{
Paraplegia
}

\section{Injuries to Elite Wheelchair Athletes}

\author{
M. S. Ferrara, MS, ATC, ${ }^{1}$ R. W. Davis, $\mathbf{P h D}^{2}$ \\ ${ }^{1}$ Pennsylvania State University, Department of Health Education, 18 White Building, \\ University Park, PA 16802, USA, ${ }^{2}$ Ball State University, Department of Physical \\ Education, University Gymnasium, Muncie, IN 47306, USA.
}

\begin{abstract}
Summary
The purpose of this project was to describe the nature, type, and frequency of athletic injuries incurred by the elite wheelchair athlete. Nineteen athletes participated in a 1-year injury recall study at an elite wheelchair training camp. An injury was defined as anything the athlete expressed concern about and (a) caused a loss of participation due to an injury or illness or (b) an injury in which a fracture, dislocation, or subluxation occurred and the athlete was able to continue participation. There were 10 male and 9 female subjects who reported their injuries from 1 fune 1987 to 31 May 1988. Fifty injuries were reported, strains and muscular injuries accounted for almost half of the injuries. Physicians were the primary care provider for $37 \%$ of the injuries, followed by physical therapists and athletic trainers at $26 \%$ and $15 \%$ respectively. Slightly over $57 \%$ of the injuries were classified as minor, missing 7 days or less of participation and 32\% were classified as major, missing 22 days or more of participation. The upper extremity was the most frequently injured, followed by the lower extremity, head and spine, and illnesses. Conventional treatments of ice, heat, modalities, and medications, were the most common methods of treating these injuries. Flexibility and strength training programmes should be implemented throughout the competitive season. Careful consideration of the training programme and workout intensity should also be evaluated.
\end{abstract}

Key words: Wheelchair Athlete; Injury; Disabled Sports; Sportmedicine.

Participation in sport and recreational activities has increased for the person with a disability in recent years. Consequently, the number of sport-related injuries have also increased. Sport and recreation are environments in which injuries must be expected and addressed forthrightly. These issues have been examined in a variety of sport settings for the athlete without a disability (Backx et al., 1989; McAuley et al., 1987; Koplan et al., 1982; Clarke and Buckley, 1980). However, few studies have attempted to quantify the time loss from participation due to an injury.

Several authors (Magnus, 1987; Bloomquist, 1986; Madorsky and Curtis, 1984) have described the common injuries suffered by the wheelchair athlete. They 
described common injuries such as abrasions, contusions, strains, sprains, and thermoregulatory disturbances and the appropriate methods for the prevention and care of these injuries. However, only Curtis and Dillon (1985) have attempted to measure and describe the frequency and types of injuries incurred by wheelchair athlete.

The National Wheelchair Athletic Association (NWAA) sponsored an elite training camp in preparation for the 1988 Olympics and Paralympics in Seoul, Korea. The purpose of the training camp was 3-fold. First, to provide an intensive training environment for athletes to improve and refine athletic performance under the direct supervision of a coach. This camp was one of the first opportunities for the athlete to work intensely with their coaches to prepare for these international competitions. Second, to provide educational sessions including the topics exercise physiology, optimal training methods to obtain peak performance, injury prevention, the effect of drugs and alcohol on sport performance, psychology of competition, and cultural insights to Korean lifestyles. The final purpose of the training camp was to conduct research on various parameters of athletic performance for the wheelchair athlete. A multidisciplinary research team examined several specific topical areas including wheelchair stroke efficiency, exercise physiology, sport psychology, and a quality of life through a health and wellness screening.

The purpose of this investigation was to establish the incidence and examine the type of athletic injuries incurred by the elite wheelchair athlete during a 1year period. The length of time the wheelchair athlete lost to practice or competition due to an injury will also be established.

\section{Methodology}

\section{Subjects}

The NWAA invited 65 athletes to the elite training camp. The roster of participants for the camp was obtained from the NWAA national office. Each participant was mailed an explanation of the injury study, an instruction booklet, and several injury survey forms prior to the camp. Each reportable injury required a separate injury form. The athletes were asked to return the completed instruments to the registration area upon arrival. The athlete was responsible for completion of the instrument.

Nineteen athletes participated in the 1-year injury recall project, 10 males and 9 females. The athletes averaged 6.4 years of experience in competitive wheelchair sports. The sample is described in terms of gender, age, and classification in Table I. The athletes were asked about their primary competitive wheelchair sport during the year. Track and field accounted for $63.6 \%$, followed by swimming $22 \cdot 7 \%$, table tennis $9 \cdot 1 \%$, and shooting $4 \cdot 5 \%$.

\section{Instrumentation}

The Wheelchair Injury Reporting System (WIRS) was the data collection instrument. The instrument was designed to obtain information relating to factors involved in an injury episode. These factors included mechanism of injury, training session in which an injury occurred, season in which the injury occurred, 
Table I Gender, age, and classification of the subjects

\begin{tabular}{lccc}
\hline Variable & Sample & Male & Female \\
\hline $\begin{array}{l}\text { Age (mean) } \\
\text { Years of experience in sports }\end{array}$ & $29 \cdot 5$ & $28 \cdot 6$ & $30 \cdot 6$ \\
(mean) & $5 \cdot 96$ & $5 \cdot 08$ & $7 \cdot 00$ \\
Classification & & & \\
IA & 3 & 2 & 1 \\
IB & 2 & 1 & 1 \\
IC & 3 & 2 & 1 \\
II & 1 & 0 & 1 \\
III & 5 & 3 & 2 \\
IV & 2 & 2 & 0 \\
V & 3 & 1 & 2 \\
\hline
\end{tabular}

competitive sport when injured, type of injury, medical professional who treated the injury, and time loss to practice/competition due to an injury. An injury was defined as anything the athlete expressed concern about which met the following conditions:

1. Loss of practice or game participation due to an injury or illness incurred which prevented participation in a sport for 1 day or more.

2. An injury in which a fracture or dislocation/subluxation occurred and the athlete was able to continue participation in the sport.

Since the secondary purpose of this investigation was to quantify the time loss from participation, minor or insignificant injuries that occurred that did not cause a time loss are not represented. In an attempt to include the significant injuries that may have occurred (fractures, dislocation, etc.) and did not cause a time loss of participation, the second definition of injury was included. The athletes were asked to report all injuries they had from 1 June 1987 to 31 May 1988. If more than 1 time loss injury occurred during this time frame, the athlete was required to complete a separate form for each injury.

The time loss definitions used by the National Athletic Injury Reporting System (NA.IRS) (Buckley and Powell, 1982) were used. A minor injury was defined as a time loss of 7 days or less from practice/competition. A significant injury was a time loss of 8-21 days and a major injury was 22 or more days lost from practice/competition. This framework allows an analysis of the nature, type, and frequency of an injury. Based on this information, preventive programmes can be designed to reduce the severity and frequency of an injury.

The year was categorised into 3 classifications, pre-season or preparation for the sport, competitive season, and off-season in which the athlete was not directly training for a sport. These classifications can identify the portion of the year in which the injury occurred.

Resulting data were described using the Statistical Package for Social Sciences (SPSSX). Due to the low number of subjects, a descriptive analysis of the injury trends and time loss factors are presented for consideration. 


\section{Results}

There were 50 injuries reported by 19 elite wheelchair athletes from 1 June 1987 to 31 May 1988. These injuries were classified according to sport, session, type of injury, treatment of the injury, the medical professional who treated the injury, mechanism of the injury, and the time loss of practice/competition due to an injury episode.

Sixty per cent of the injuries occurred in those involved in track and field and $20 \%$ in swimming events. The competitive season accounted for $48 \%$ of the reported injuries and $62 \%$ of these injuries occurred during a practice session. Sixty five per cent of the reported injuries were identified as acute or sudden onset injuries while $23 \%$ of the reported injuries were overuse or a gradual onset of signs and symptoms. Only $12 \%$ of the reported injuries were not related to athletics or sport participation. Several of these non-sport related injuries occurred during wheelchair transfer or transport occasions.

Strains and muscular injuries accounted for almost half $(48 \%)$ of the injuries

Table II The types of injuries reported by the subjects during a 1-year period

\begin{tabular}{lrc}
\hline Type of injury & N & Percentage \\
\hline Strain & 24 & 48 \\
Abrasion & 11 & 22 \\
Contusion & 5 & 10 \\
Blisters & 3 & 6 \\
Fracture & 3 & 6 \\
Sprain & 2 & 4 \\
Laceration & 1 & 2 \\
Illness & 1 & 2 \\
\hline
\end{tabular}

and abrasions accounted for $22 \%$ as shown in Table II. The upper extremity was the most commonly injured area, accounting for $58 \%$ of the total injuries followed by the lower extremity, $22 \%$, neck and spine $18 \%$, and $2 \%$ were related to illness. Within the upper extremity, the shoulder and wrist were the most commonly

Table III The number of injuries to the upper extremity

\begin{tabular}{lcc}
\hline Body part & N & Percentage \\
\hline Shoulder & 8 & $27 \cdot 6$ \\
Wrist & 6 & $20 \cdot 7$ \\
Fingers & 5 & $17 \cdot 2$ \\
Hand & 4 & $13 \cdot 8$ \\
Elbow & 4 & $13 \cdot 8$ \\
Upper arm & 1 & $3 \cdot 4$ \\
Lower arm & 1 & $3 \cdot 4$ \\
\hline
\end{tabular}

injured area in these athletes as noted in Table III. There did not appear to be a dominant side of the body that was involved in the injury episode. In fact, bilateral 
injuries had the second greatest frequency of $32 \%$, compared to the left extremity $34 \%$, right $28 \%$, and neither $4 \%$.

Physicians were the primary care provider for $37 \%$ of the injuries, followed by physical therapist and athletic trainers at $26 \%$ and $15 \%$ respectively. However, $12 \cdot 2 \%$ of the injuries were taken care of by the athlete without seeking professional assistance. It is interesting to note that the athletes in the study reported that the coach was the primary care provider of their injuries only $2 \%$ of the time.

The athletes were asked to indicate the form of treatment they received for the injury. This was placed on a continuum from surgery, to hospitalisation, to immobilisation, to medication, to modalities, to protective padding, representing the most severe to least severe type of treatment. In general, the treatment of these injuries followed the same conventional treatment patterns as the athlete without a disability. Thirty per cent of the injuries were treated with modalities such as ice, heat, or an electrical modality. Medications such as anti-inflammatories and analgesics were involved $25 \%$ of the time.

Analysis of the mechanism of injury variable indicate that direct impact with the floor, another chair, or another object was the most common method of injury for $49 \%$ of the injuries. Repetitive stress or overuse accounted for $35 \%$ of the injuries. A direct impact mechanism generally results in an acute injury such as a fracture, contusion, or soft tissue injury. Moreover, many of the overuse injuries may not have been reported since they did not cause a time loss to practice/competition. The athlete may have been able to continue participation in sport even though he/she was participating at submaximal levels.

The amount of time the athlete misses from training or competition is vital. The athlete needs to continue to participate in order to maintain the optimal physiological levels needed for competition. The time loss to practice/competition for the

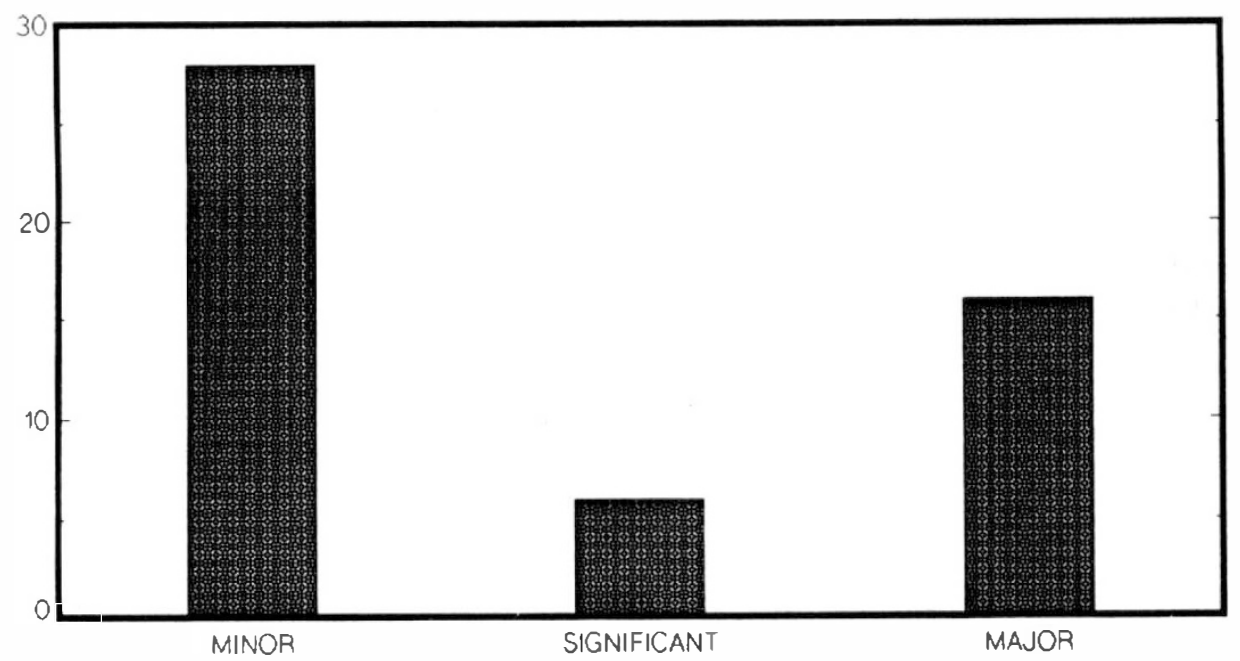

Figure Time loss to an injury/illness.

wheelchair athlete is reported in the Figure. Minor injuries (missing 7 days or less) accounted for $57 \%$ of the injuries. However, an alarmingly high percentage $(32 \%)$ of the injuries were classified as major (missing 22 days or more). 


\section{Discussion}

The purpose of this study was to describe the factors relating to an injury and the time loss of participation due to an injury for the elite wheelchair athlete. The subjects participated in a 1-year injury recall study.

The results of this study concur with the results reported by Curtis and Dillon (1985). They reported that soft tissue injuries such as sprains and strains accounted for $33 \%$ of the injuries, and abrasions accounted for $26 \%$ of the injuries. Further, $70 \%$ of the injuries occurred in track, road racing, or basketball events. The participants in the 21-30 years age group suffered the highest number of injuries when compared to other age groups. This investigation found that strains were the most common type of reported injury.

There are several important interrelationships to be considered by the physician, physical therapist, athletic trainer, and coach in designing prevention programmes. The athletes related that changes in their training techniques and patterns may have precipitated their injury. They related such mechanisms as: 'radically altering my seat position in the chair', 'learning a new propulsion technique for a prolonged period of time', and 'getting my hand and fingers caught in the spokes of the wheel'. When teaching or learning new techniques, gradually increase the intensity of the activity to allow the body to adjust to the new demands of the technique. This will allow a new set of muscles to become accustomed to the demands of the new activity.

Several athletes were also injured in non-sport related events. Three of these athletes were hurt in chair transfer, 2 of the athletes suffered a fracture. All individuals involved in wheelchair athletics should be properly taught and trained in the transferring technique of a wheelchair athlete. Additionally, wheelchair athletes should be able to educate and explain the transfer technique to people who are not familiar with the transfer technique. This should help to reduce the number of injuries that are not sport-related.

Conventional treatment regimens seem to be very effective. Furthermore, when rehabilitating the athlete with a disability, the goal of the physician and therapist should be to restore the athlete to competitive form. These athletes are highly skilled and need to achieve competitive physiological condition as quickly and safely as possible. The use of multiple treatment modalities and strengthening devices would be beneficial in the rehabilitation of the elite wheelchair athlete. Therefore, an aggressive approach should be taken by the medical profession.

Prevention programmes should include a comprehensive approach in which several components of fitness are addressed. These include muscular strength, muscular endurance, cardiovascular endurance, and flexibility. Perhaps the component most overlooked is flexibility. Slightly less than $50 \%$ of these athletes reported strains and muscular injuries. A supervised flexibility programme would seem to be indicated. Further, exercise prescription must address the upper extremity and in particular the shoulder complex since $16 \%$ of the athletes reported an injury to this area. Weight training programmes must be developed and supervised to reduce the rate of injury reported to the shoulder. 


\section{Conclusions}

This was a first attempt to identify the time loss factor to practice/competition due to injury or illness. The findings of this study indicate that the wheelchair athlete appears to have the same type and frequency of injury as the athlete without a disability. The time loss factor appears to be higher for major injuries as compared to the athlete without a disability. This could be due to the delayed healing process of the athlete with a spinal cord injury as well as a conservative treatment approach.

A comprehensive team approach needs to be utilised to reduce the rate and severity of injuries to the wheelchair athlete. Physicians, athletic trainers, and physical therapists should be involved in providing a physical screening assessment of the athlete to determine if any musculoskeletal deficiencies are present that may predispose the athlete to injury. This assessment should consist of flexibility, strength, cardiovascular condition, and a physical examination performed by a physician. Based on the assessment, corrective and preventive programmes can be designed to reduce the rate and severity of injury. A longitudinal epidemiological prospective study should be performed to measure the actual rate and risk of injury using conventional scientific applications.

\section{Acknowledgement}

The authors would like to thank the NWAA and the athletes for their participation in this project, Dr W. E. Buckley for his review of the manuscript and Dr Van Nelson for his statistical assistance.

\section{References}

BACKX FJ, ERICH WB, Kemper AB, VerbeEk AL 1989 Sports injuries in school-aged children. American fournal of Sports Medicine 17:234-240.

BLOOMQUIST LE 1986 Injuries to athletes with physical disabilities: Prevention implications. The Physician and Sportsmedicine 14:97-105.

BUCKLEY WE, POWELL JP 1982 NAIRS an epidemiological overview of the severity of injury in college football 1975-1980 seasons. Athletic Training 17:279-282.

ClaRke KS, BUCKLEY WE 1980 Women's injuries in collegiate sports. The American fournal of Sports Medicine 8:187-191.

CurTis KA, Dillon DA 1985 Survey of wheelchair athletic injuries: Common patterns and prevention. Paraplegia 23:170-175.

Koplan JP, Powell KE, Sikes RK, ShiRley RW, Campbell CC 1982 An epidemiologic study of the benefits and risks of running. Fournal of the American Medical Association 248:3118-3121.

MADORSKY JG, CURTIS KA 1984 Wheelchair sports medicine. The American Fournal of Sports Medicine 12:128-132.

MAGNUS BC 1988 Medical care for wheelchair athletes. Adapted Physical Activity Quarterly 5:90-95.

MCAulfy E, HudASH G, ShiElds K, et al. 1987 Injuries in women's gymnastics. The American fournal of Sports Medicine 15:558-565. 